\title{
Entrevistas realizadas a distância no campo da pesquisa qualitativa em saúde ${ }^{1}$
}

\author{
Camila da Silva Gonçalo ${ }^{a}$, Nelson Filice de Barros ${ }^{b}$ \\ ${ }^{a}$ Doutora em Saúde Coletiva, Programa de Pós-graduação em Saúde Coletiva, Universidade Estadual de \\ Campinas, Campias, SP, Brasil \\ ${ }^{\text {b }}$ Doutor em Saúde Coletiva, Programa de Pós-graduação em Saúde Coletiva, Universidade Estadual de \\ Campinas, Campias, SP, Brasil, Docente da Faculdade de Ciências Médicas, Universidade Estadual de \\ Campinas - Unicamp, Campinas, SP, Brasil
}

\begin{abstract}
Resumo: O objetivo do artigo é apresentar e discutir os resultados de uma revisão sistemática da literatura referente ao uso de entrevistas realizadas a distância (ED) nas pesquisas qualitativas em saúde. Foram conduzidas buscas na PubMed e CINAHL, sendo incluídas 37 referências. Os dados foram organizados segundo autoria, ano de publicação, título do periódico e objetivo do manuscrito. A análise dos resultados revelou que o assunto mais recorrente foi a comparação das ED com outros métodos de coleta de dados. Baixo custo, maior facilidade no acesso aos entrevistados, maiores taxas de participação e recrutamento foram apontados como as principais vantagens proporcionadas pelas ED. As ED foram utilizadas para vários fins, desde a validação de instrumentos de coleta de dados até para fins diagnósticos. Conclui-se que há necessidade da ampliação da discussão sobre o tema, pois a incorporação de tecnologias da informação e comunicação no campo da saúde representa uma via de acesso valiosa na captação de dados qualitativos.
\end{abstract}

Palavras-chave: Pesquisa Qualitativa, Entrevistas como Assunto, Entrevista, Coleta de Dados.

\section{Distance interviews in the field of qualitative health research}

\begin{abstract}
In this study, we aimed to present and discuss the results of a systematic literature review on the use of Distance Interviews (DI) in qualitative health research. Searches were conducted in the PubMed and CINAHL databases and 37 references were included. Data were organized according to author, year of publication, journal title, and manuscript objective. The results revealed that the most recurrent theme was the comparison of Distance Interviews with other methods of data collection. Low cost, easier access to respondents, higher participation rates, and recruitment of volunteers were identified as the main benefits offered by DI. Distance Interviews were used for various purposes, from validation of instruments for data collection to diagnosis. We concluded that there is a need to expand on the theme explored in this article, because the incorporation of information and communication technologies in the healthcare field represents a valuable access route in capturing qualitative data.
\end{abstract}

Keywords: Qualitative Research, Interviews as Topic, Interview, Data Collection.

\section{Introdução}

Inovações nos sistemas de informação têm imprimido um novo rumo na comunicação em praticamente todas as áreas do conhecimento, inclusive na área da saúde. A variedade de tipos de sistemas e aparatos para efetivar a troca de um conjunto de dados, o aumento na velocidade dessas trocas e seu maior dinamismo vêm sendo apontados como as principais características relacionadas 
com o cenário das tecnologias da informação e comunicação em saúde (CARR; WORHT, 2001; PACHECO et al., 2005).

A integração dessas tecnologias na investigação qualitativa tem proporcionado aos pesquisadores $\mathrm{da}$ área novas oportunidades e abordagens para captação de dados (BAMPTON; COWTON, 2002; JAMES, 2007). Nesse sentido, a literatura revela que a incorporação do uso de entrevistas via telefone e e-mail na pesquisa qualitativa em saúde é um fato pertinente (MANN; STEWART, 2000; ASELTON, 2012), principalmente porque vários autores identificaram que as entrevistas realizadas a distância (ED) têm apresentado características relevantes, tais como a redução do tempo de entrevista, o favorecimento de maior taxa de resposta (EVANS et al., 2004; PINTO-MEZA et al., 2005; FEVEILLE; OLSEN; HOGH, 2007; ROCHELEAU et al., 2012) e a reduçáo de custos (LANG et al., 2011; JOHANSEN; WEDDERKOPP, 2010; NAGELHOUT et al., 2010; WARD-KING et al., 2010).

Considerando que o telefone e o e-mail podem ser recursos tecnológicos úteis para investigaçóes científicas e que a condução de entrevistas realizadas a distância (ED) é um assunto pouco explorado, o presente artigo tem como objetivo apresentar e discutir os resultados de uma revisão sistemática da literatura referente ao uso de entrevistas realizadas a distância (ED) na pesquisa qualitativa em saúde.

\section{Métodos}

O principal fator motivador para o desenvolvimento da presente revisão foi a necessidade de realizar pesquisas que pudessem abranger populaçóes situadas em áreas mais longínquas, como, por exemplo, em estudos de abrangência nacional. Ressalta-se que os autores do artigo são pesquisadores da área de ciências sociais aplicadas à saúde, posição que demanda a busca constante por interfaces com as diferentes áreas do conhecimento. Considerando o cenário supracitado, os autores têm encontrado dificuldades na obtenção de referências que ofereçam subsídios para o planejamento e execução de ED. A reflexão sobre esse contexto gerou a pergunta norteadora do presente estudo: "Qual é o cenário do uso de ED na pesquisa qualitativa em saúde?”. Para tanto, foi feita uma pesquisa prévia das principais fontes de informaçáo científica no campo da saúde. Constatou-se que a PubMed e a CINAHL têm sido as bases de dados identificadas como as mais relevantes nesse campo (EVANS, 2002; FALAGAS et al., 2008; ANDERS; EVANS, 2010).

Com a finalidade de localizar o leitor nos métodos empregados para a revisáo de literatura realizada, bem como de suscitar a busca de interfaces com outras áreas, segue uma breve descrição das bases de dados utilizadas:

- PubMed: Compreende mais de 22 milhóes de citaçóes da literatura biomédica, além de revistas e livros on-line. As referências disponibilizadas nessa base de dados podem incluir links para conteúdo de texto completo (www.pubmed.org);

- CINAHL: Contém mais de 1 milhão de referências relacionadas à enfermagem, medicina alternativa/complementar, biomedicina, saúde do consumidor, biblioteconomia de ciências da saúde e outras 17 disciplinas no campo da saúde. A base de dados disponibiliza acesso a livros, dissertações, atas de conferências, software educacional, materiais audiovisuais e capítulos de livros. $\mathrm{O}$ acesso à base de dados CINAHL é feito por meio da plataforma EBSCOHOST (http://www.ebscohost.com), que oferece mecanismos de busca e acesso a diferentes áreas do conhecimento, tais como: arte e arquitetura; comunicação e mídia; engenharia; teoria e sistemas de computadores; novas tecnologias e contexto social/profissional; odontologia; economia; educação; história do mundo; ciências humanas; ciências políticas; esporte e medicina esportiva.

Em 12 de janeiro de 2013, foram conduzidas quatro buscas distintas na PubMed e CINAHL. Ressalta-se que a primeira base de dados foi acessada a partir de sua página da internet (www.pubmed. org) e a segunda base de dados foi acessada via EBSCOHOST (www.ebscohost.com).

Os termos de busca utilizados foram: Telephone Interview; Telephone Interviewing; E-mail Interview; E-mail Interviewing. Não houve restrição de idioma, tampouco de período de publicação. Essas estratégias foram aplicadas visando ampliar o universo das buscas.

Foram incluídos somente estudos qualitativos disponíveis on-line, na versão full text, independente da data de publicação, do idioma e que de fato relatassem a coleta dos dados por meio de entrevista a distância. Em contrapartida, foram excluídas as referências em duplicatas, bem como as não disponíveis on-line e na íntegra. As buscas foram conduzidas por dois pesquisadores independentes, separadamente nas duas bases de dados, porém na mesma data. Ressalta-se que todo o procedimento de busca e análise sistemática dos dados foi realizado segundo os critérios propostos por Gonçalo et al. (2012). 


\section{Resultados e discussão}

Ao todo foram incluídas 37 referências, sendo que a maioria dessas versou a respeito de entrevistas telefônicas $(\mathrm{n}=35)$, e 2 referências abordaram o tema das entrevistas por internet (e-mail).

O levantamento bibliográfico foi realizado sem restrição de data de publicação, fato que possibilitou identificar como artigo mais antigo o trabalho de Mooney, Pollack e Corsa Junior (1968) divulgado em 1968, publicado no Public Health Reports e que se encontra disponível para download gratuito na base de dados PubMed. Em contrapartida, os artigos mais recentes encontrados foram localizados pela CINAHL (LI et al., 2012; ROCHELEAU et al., 2012; GUSTAFSSON; ERICSSON; ANDERSSON, 2012) e os periódicos que publicaram tais estudos foram, respectivamente: Nursing Science \& Research in the Nordic Countries; BMC Public Health; International Journal of Public Health. Os dois últimos títulos referem-se a periódicos específicos e, desse modo, é possível identificar algumas conexões entre o uso das ED com as pesquisas em saúde pública (NAGELHOUT et al., 2010; LI et al., 2012). A Tabela 1 ilustra as principais características dos estudos incluídos (Figura 1).

\subsection{Comparação entre as diferentes formas de utilização das ED}

$\mathrm{O}$ assunto mais recorrente encontrado foi a comparação de ED com outras técnicas de coleta de dados (HUANG, 2003; FONG et al., 2009; SIKORSKII et al., 2009; JOHANSEN; WEDDERKOPP, 2010; NAGELHOUT et al.,
2010; LANG et al., 2011; LI et al., 2012; ROCHELEAU et al., 2012). Nesse contexto, características interessantes foram encontradas, como no estudo de Rocheleau et al. (2012), que mostra as evidências de entrevistas telefônicas concedidas por pessoas detentoras de números de telefones fixos bem estabelecidas; em contrapartida, as entrevistas realizadas por meio de telefones celulares apresentam desafios para a condução das pesquisas no que tange à falta de privacidade ao conceder entrevistas, quando o indivíduo se encontra em locais que náo permitem salvaguardar sua intimidade. Os autores verificaram que a coleta de dados por carta foi substancialmente superior às entrevistas telefônicas nas taxas de participação e recrutamento de voluntários. No entanto, a captação de dados por meio das entrevistas telefônicas mostrou-se mais favorável do que a entrevista por carta, no esclarecimento das respostas para perguntas complexas ou requisitos de elegibilidade.

Johansen e Wedderkopp (2010) compararam as entrevistas conduzidas por Short Message Service (SMS) com as entrevistas telefônicas e relatam que a primeira tecnologia apresenta uma vantagem no monitoramento e confiabilidade dos dados, uma vez que estes ficam registrados nos servidores que armazenam tais informaçóes. Além disso, a tecnologia SMS possibilita o envio de uma única mensagem curta para um grupo de pessoas, independentemente do tempo, lugar ou sua fixação, reduzindo o custo e o tempo considerado na condução das entrevistas.

Lang et al. (2011) ressaltam que, dependendo da faixa etária dos entrevistados, o uso das entrevistas telefônicas não oferece respostas tão claras quanto às obtidas por meio de questionário autoaplicado e entrevista face a face.

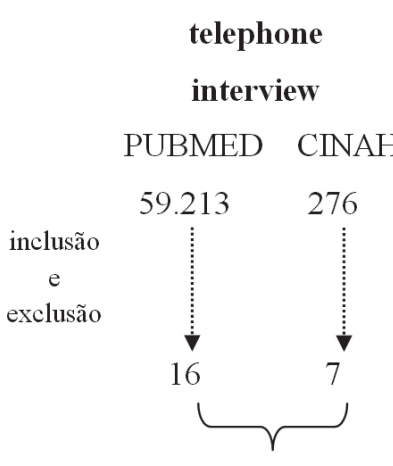

23

\section{telephone}

interviewing

PUBMED CINAHL

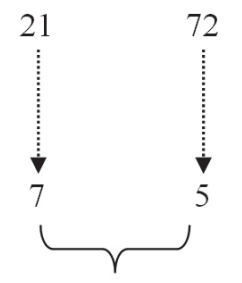

12 email

interview

PUBMED CINAHL

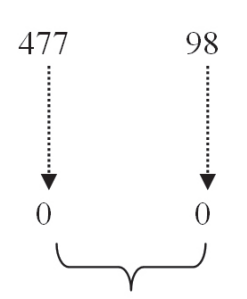

0 email

\section{interviewing}

PUBMED CINAHL

37 referências incluídas e analisadas

(24 PUBMED + 13 CINAHL)

Figura 1. Resultados das buscas conduzidas na base de dados PubMed e CINAHL. 


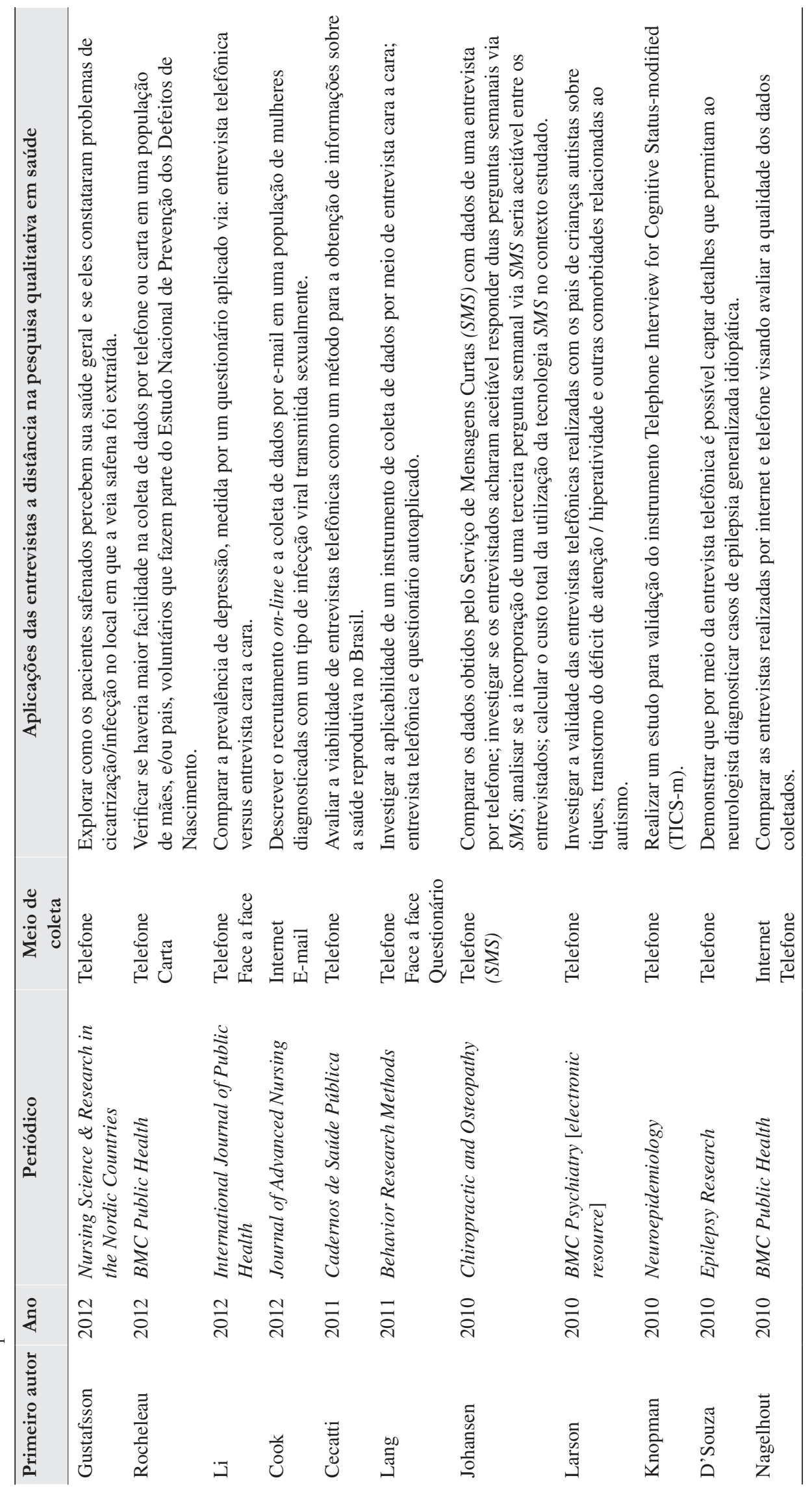




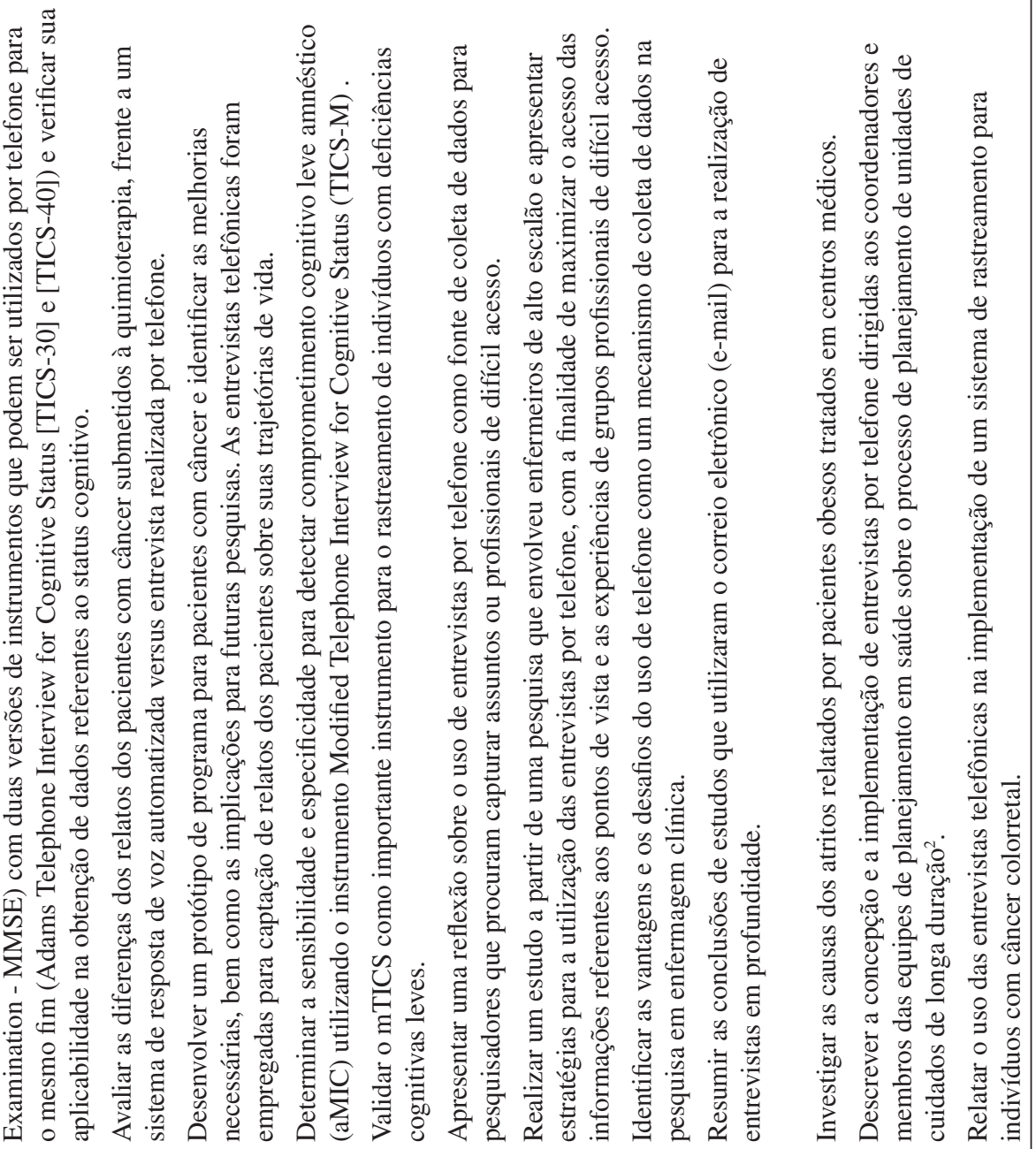

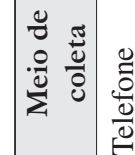

$\frac{\mathscr{0}}{\frac{0}{0}} \frac{\frac{0}{0}}{\frac{0}{0}}$

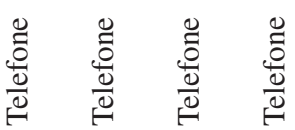

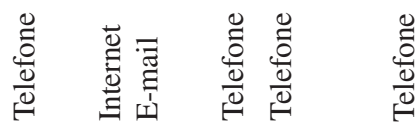

है

$\cong$

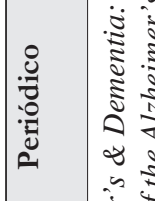

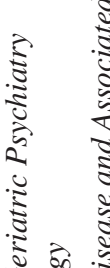

$\stackrel{3}{3}$

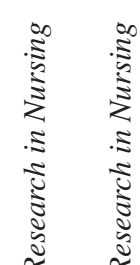

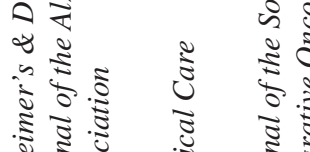

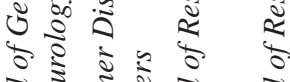

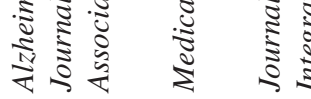

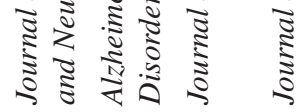

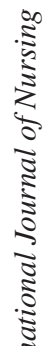

$\stackrel{乛}{\Xi}$

莺

ठे ठ․

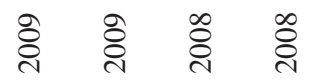

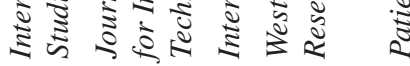

¿

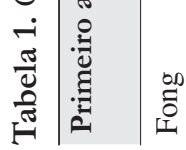

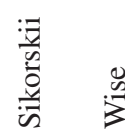

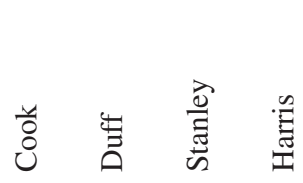

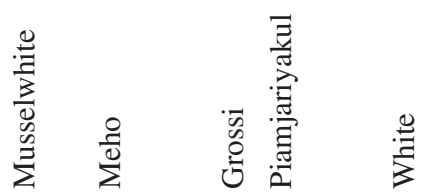




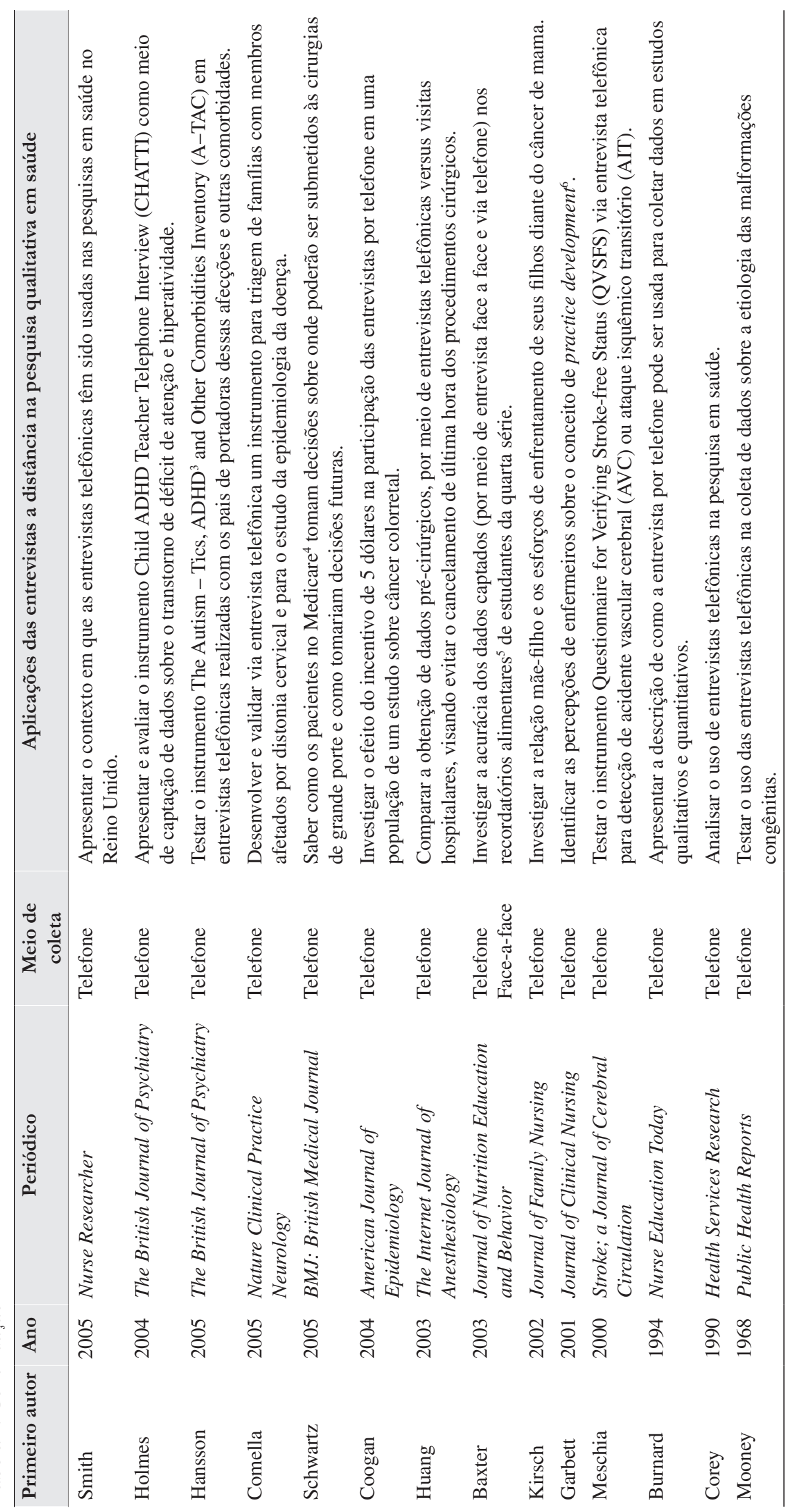


Nagelhout et al. (2010) comentam que foram verificadas diferenças entre dados coletados por entrevistas realizadas via internet e via telefone, mas estas são mínimas, não sendo favoráveis à primeira, tampouco à segunda forma de entrevista.

\subsection{Viabilidade das formas de utilização das ED}

A viabilidade das ED (MOONEY; POLLACK; CORSA JUNIOR, 1968; MUSSLEWHITE et al., 2007; HARRIS et al., 2008; STANLEY, 2008; D'SOUZA et al., 2010; CECATTI et al., 2011) e a avaliação de instrumentos para captação de dados por telefone também foram temas que sobressaíram nos resultados da presente análise (COREY; FREEMAN, 1990; HOLMES et al., 2004; HANSSON et al., 2005; COMELLA, 2005). Nesse contexto, Cecatti et al. (2011) afirmaram que o uso das entrevistas telefônicas se mostrou viável para obtenção de informaçôes de saúde reprodutiva entre mulheres, especialmente quando a entrevista abordou eventos relativamente recentes, e nos casos em que as entrevistadas disponibilizaram aos pesquisadores mais de um número de telefone.

D'Souza et al. (2010) testaram o emprego de entrevistas telefônicas como alternativa útil em estudos epidemiológicos de epilepsia. Os autores verificaram que as entrevistas telefônicas proporcionaram concordância quase perfeita no diagnóstico de convulsóes, epilepsia e epilepsia generalizada idiopática.

As entrevistas telefônicas mostraram-se viáveis para atingir assuntos e indivíduos de difícil acesso (STANLEY, 2008; HARRIS et al., 2008). Além disso, Musselwhite et al. (2007) propuseram-se a identificar as vantagens e os desafios de usar o telefone como um mecanismo de coleta de dados na pesquisa em enfermagem clínica. Os autores concluíram que é necessário haver um treinamento para os entrevistadores a fim de estabelecer uma comunicação eficaz entre eles e os participantes da pesquisa. Também destacam que a realização de procedimentos padronizados de acompanhamento é necessária para assegurar o êxito da coleta de dados via telefone.

Mooney, Pollack, Corsa Junior (1968) concluíram que a captação de informaçôes por entrevista telefônica é uma ação viável e apresenta-se de baixo custo quando comparada com outros procedimentos de coleta de dados.

\subsection{Instrumentos utilizados nas ED}

Referente à avaliação de instrumentos aplicados nas ED, foi verificada uma variedade de temas e procedimentos, predominando o uso das entrevistas telefônicas, como pode ser observado nos parágrafos que seguem.

Hansson et al. (2005) concluíram que The Autism-Tics, ADHD and Other Comorbidities Inventory $(A-T A C)$ parece ser um instrumento válido e confiável na identificação de transtornos relacionados ao autismo, transtorno do déficit de atenção com hiperatividade, tiques, distúrbios de aprendizagem e transtornos do desenvolvimento da coordenação.

Comella (2005) testaram o instrumento Beth Israel Dystonia Screen (BIDS) e destacaram sua utilidade em estudos genéticos de distonia cervical, porém os autores alertam que é necessária uma validação adicional antes da aplicação desse instrumento em estudos epidemiológicos.

Holmes et al. (2004) relataram que Child ADHD Teacher Telephone Interview (CHATTI) se mostrou uma ferramenta promissora para avaliar os sintomas de transtorno do déficit de atenção com hiperatividade (TDAH) no ambiente escolar e pode ser útil tanto na clínica quanto em pesquisas.

\subsection{Concentração de publicações a respeito das ED}

Verificou-se uma concentração de artigos publicados nos periódicos BMC Public Health $(\mathrm{n}=2)$; Journal of Research in Nursing $(\mathrm{n}=2)$; e The British Journal of Psychiatry $(\mathrm{n}=2)$, sendo que a maior parte dos artigos analisados foi publicada em revistas da área de enfermagem $(n=8)$ e os períodos que apresentaram maior frequência de publicaçôes das entrevistas realizadas a distância foram os dos anos de 2009 ( $n=5)$ e 2010 ( $n=5)$.

Embora a maior parte das referências analisadas tenha sido localizada pela PubMed $(\mathrm{n}=24)$, a CINAHL identificou 13 artigos citados na presente revisão.

\subsection{Limitações do estudo}

Alguns periódicos pesquisados encontram-se indexados nas duas bases de dados utilizadas na presente revisão, porém a análise dos dados revelou uma inconsistência, na medida em que, na data do levantamento, alguns artigos foram recuperados somente pela CINAHL. Foi observada uma 
concentração de estudos publicados em periódicos de enfermagem. Tal constataçẫo ocorreu em função do levantamento conduzido na base de dados CINAHL, pois a PubMed recuperou apenas uma dessas referências. Nesse contexto, sugere-se que futuras revisóes sistemáticas do tema das ED sejam conduzidas em outras bases de dados, a fim de esclarecer se esses achados foram introduzidos pela pesquisa na CINAHL ou se, de fato, a produção de artigos que abordam essa temática tem sido maior na área de enfermagem.

\section{Considerações finais}

Nas bases pesquisadas e manuscritos analisados, o assunto mais recorrente foi a comparação de ED (entrevistas por telefone, entrevistas por e-mail, entrevistas por carta, entrevistas por SMS, entrevistas por internet) com outras técnicas de coleta de dados (entrevistas face a face e questionários autoaplicados), seguido de estudos de viabilidade das ED e de estudos avaliativos de instrumentos para captação de dados por telefone.

Dentre as técnicas de ED encontradas na presente revisão, a entrevista telefônica foi a mais recomendada, principalmente devido ao seu baixo custo; a sua capacidade de abordar assuntos e pessoas de difícil acesso e a economia de tempo na conduçáo das entrevistas.

\section{Referências}

ANDERS, M. E.; EVANS, D. P. Comparison of PubMed and Google Scholar Literature Searches. Respiratory Care, Dallas, v. 55, n. 5, p. 578-583, 2010.

ASELTON, P. Using the Internet for Qualitative Research in Nursing. Open Access Scientific Reports, New York, v. 1, n. 126, p. 1, 2012.

BAMPTON, R.; COWTON, C. J. The E-Interview. $F Q S$ : Forum Qualitative Social Research, Berlim, v. 3, n. 2, p. 17-29, 2002.

BAXTER, S. D. et al. Accuracy of fourth-graders' dietary recalls of school breakfast and school lunch validated with observations: in-person versus telephone interviews. Journal of Nutrition Education and Behavior, New York, v. 35, n. 3, p. 124-34, 2003. http://dx.doi.org/10.1016/ S1499-4046(06)60196-6

BURNARD, P. The telephone interview as a data collection method. Nurse Education Today, Edinburgh, v. 14, n. 1, p. 67-72, 1994. http://dx.doi. org/10.1016/0260-6917(94)90060-4

CARR E, WORTH, C. J. The use of the telephone interview for research. Nursing Times Research, London, v. 6, n. 1, p. 511-52, 2001. http://dx.doi. org/10.1177/136140960100600107
CECATTI, J. G. et al. Computer-assisted telephone interviewing (CATI): using the telephone for obtaining information on reproductive health. Cadernos de Saude Publica, Rio de Janeiro, v. 27, n. 9, p. 1801-8, 2011.

COMELLA, C. L. Can families be screened for cervical dystonia using a telephone interview? Nature Clinical Practice Neurology, London, v. 2, n. 1, p. 20-1, 2005. PMid:16932515. http://dx.doi.org/10.1038/ncpneuro0073 COOGAN, P. F.; ROSENBERG, L. Impact of a financial incentive on case and control participation in a telephone interview. American Journal of Epidemiology, Baltimore, v. 160 , n. 3, p. $295-8,2004$. PMid:15258003. http:// dx.doi.org/10.1093/aje/kwh190

COOK, C. Email interviewing: generating data with a vulnerable population. Journal of Advanced Nursing, Oxford, v. 68, n. 6, p. 1330-9, 2012. PMid:21999402. http://dx.doi.org/10.1111/j.1365-2648.2011.05843.x

COOK, S. E.; MARSISKE, M.; McCOY, K. J. The use of the Modified Telephone Interview for Cognitive Status (TICS-M) in the detection of amnestic mild cognitive impairment. Journal of Geriatric Psychiatry and Neurology, Thousand Oaks, v. 22, n. 2, p. 103-9, 2009. PMid:19417219 PMCid:PMC2913129. http://dx.doi. org/10.1177/0891988708328214

COREY, C. R.; FREEMAN, H. E. Use of telephone interviewing in health care research. Health Services Research, Chicago, v. 25, n. 1, p. 129-44, 1990.

D'SOUZA, W. J. et al. The use of computer-assistedtelephone-interviewing to diagnose seizures, epilepsy and idiopathic generalized epilepsy. Epilepsy Research, Amsterdam, v. 91, n. 1, p. 20-7, 2010. PMid:20656458. http://dx.doi.org/10.1016/j.eplepsyres.2010.06.003

DUFF, K.; BEGLINGER, L. J.; ADAMS, W. H. Validation of the modified telephone interview for cognitive status in amnestic mild cognitive impairment and intact elders. Alzheimer Disease and Associated Disorders, Hagerstown, v. 23, n. 1, p. 38-43, 2009. PMid:18695589 PMCid:PMC2649962. http://dx.doi. org/10.1097/WAD.0b013e3181802c54

EVANS, D. Database searches for qualitative research. Journal of the Medical Library Association, Chicago, v. 90, n. 3, p. 290-3, 2002.

EVANS, M. et al. Assessing mental health in primary care research using standardized scales: can it be carried out over the telephone? Psychological Medicine, Philadelphia, v. 34, p. 157-162, 2004. http://dx.doi.org/10.1017/ S0033291703008055

FALAGAS, M. E. et al. Comparison of PubMed, Scopus, Web of Science, and Google Scholar: strengths and weaknesses. FASEB Journal, Bethesda, v. 22, n. 2, p. 338-42, 2008. PMid:17884971. http://dx.doi. org/10.1096/fi.07-9492LSF

FEVEILLE, H.; OLSEN, O.; HOGH, A. A randomized trial of mailed questionnaires versus telephone interviews: Response patterns in a survey. BMC Medical Research Methodology, London, v. 7, n. 27, p. 1-7 2007. 
FONG, T. G. et al. Telephone interview for cognitive status: Creating a crosswalk with the Mini-Mental State Examination. Alzheimer's \& Dementia, Orlando, v. 5, n. 6, p. 492-7, 2009. PMid:19647495 PMCid:PMC2783323. http://dx.doi.org/10.1016/j.jalz.2009.02.007

GARBETT, R.; McCORMACK, B. The experience of practice development: an exploratory telephone interview study. Journal of Clinical Nursing, Boston, v. 10, n. 1, p. 94-102, 2001. http://dx.doi. org/10.1046/j.1365-2702.2001.00455.x

GONÇALO, C. S. et al.Planejamento e execução de revisóes sistemáticas da literatura. Brasília Médica, Brasília, v. 49, n. 2, p. 104-110, 2012.

GROSSI, E. et al. Complexity of attrition in the treatment of obesity: clues from a structured telephone interview. International Journal of Obesity, London, v. 30, n. 7, p. 1132-7, 2006. PMid:16432536. http://dx.doi. org/10.1038/sj.ijo.0803244

GUSTAFSSON, A. S.; ERICSSON, T.; ANDERSSON, $S$. The occurrence of problems in connections with the saphenous vein harvest surgical site? A telephone interview project three months after Coronary Artery Bypass. Nursing Science \& Research in the Nordic Countries, Oslo, v. 32, n. 1, p. 41-43, 2012.

HANSSON, S. L. et al. Psychiatric telephone interview with parents for screening of childhood autism - tics, attention-deficit hyperactivity disorder and other comorbidities (A-TAC): preliminary reliability and validity. British Journal of Psychiatry, London, v. 187, p. 262-7, 2005. PMid:16135864. http://dx.doi.org/10.1192/ bjp.187.3.262

HARRIS, L. et al. Accessing elite nurses for research: reflections on the theoretical and practical issues of telephone interviewing. Journal of Research in Nursing, London, v. 13, n. 3, p. 236-248, 2008. http://dx.doi. org/10.1177/1744987107084671

HOLMES, J. et al. The Child Attention-Deficit Hyperactivity Disorder Teacher Telephone Interview (CHATTI): reliability and validity. British Journal of Psychiatry, London, v. 184, p. 74-8, 2004. http://dx.doi. org/10.1192/bjp.184.1.74

HUANG, J. Comparison of the Effectiveness of Different Preoperative Screening Methods: Telephone Interview versus Hospital Visit. Internet Journal of Anesthesiology, Houston, v. 7, n. 2, p. 26, 2003. Disponível em: http:// ispub.com/IJA/7/2/11447.

JAMES, $N$. The use of email interviewing as a qualitative method inquiry in educational research. British Educational Research Journal, London, v. 33, n. 6, p. 963-967, 2007.

JOHANSEN, B.; WEDDERKOPP, N. Comparison between data obtained through real-time data capture by $S M S$ and a retrospective telephone interview. Chiropractic \& Osteopathy, London, v. 18, p. 10, 2010. PMid:20500900 PMCid:PMC2883994. http://dx.doi. org/10.1186/1746-1340-18-10

KIRSCH, S. E. D.; BRANDT, P. A. Telephone Interviewing: A Method to Reach Fathers in Family
Research. Journal of Family Nursing, Thousand Oaks, v. 8, n. 1, p. 73-84, 2002.

KNOPMAN, D. S. et al. Validation of the telephone interview for cognitive status-modified in subjects with normal cognition, mild cognitive impairment, or dementia. Neuroepidemiology, New York, v. 34, n. 1, p. 34-42, 2010. PMid:19893327 PMCid:PMC2857622. http://dx.doi. org/10.1159/000255464

LANG, F. R. et al. Short assessment of the Big Five: robust across survey methods except telephone interviewing. Behavior Research Methods, New York, v. 43, n. 2, p. 548-67, 2011. PMid:21424189 PMCid:PMC3098347. http://dx.doi.org/10.3758/s13428-011-0066-z

LARSON, T. et al. The autism--tics, AD/HD and other comorbidities inventory (A-TAC): further validation of a telephone interview for epidemiological research. BMC Psychiatry, London, v. 7, n. 10, p:1, 2010. PMid:20055988 PMCid:PMC2823676. http://dx.doi. org/10.1186/1471-244X-10-1

LI, C. et al. A comparison of depression prevalence estimates measured by the Patient Health Questionnaire with two administration modes: computer-assisted telephone interviewing versus computer-assisted personal interviewing. International Journal of Public Health, New York, v. 57, n. 1, p. 225-33, 2012. PMid:21523617. http:// dx.doi.org/10.1007/s00038-011-0253-9

MANN, C.; STEWART, F. Internet Communication and Qualitative Research: A Handbook for Researching Online. London: Sage, 2000. p.65-97. PMid:10761725.

MEHO, L. I. E-Mail Interviewing in Qualitative Research: A Methodological Discussion. Journal of the American Society for Information Science and Technology, New York, v. 57, n. 10, p. 1284-1295, 2006. http://dx.doi. org/10.1002/asi.20416

MESCHIA, J. F. et al. Verifying the stroke-free phenotype by structured telephone interview. Stroke, Dallas, v. 31, n. 5, p. 1076-80, 2000.

MOONEY, H. W.; POLLACK, B. R.; CORSA JUNIOR, L. Use of telephone interviewing to study human reproduction. Public Health Reports, Washington, v. 83, n. 12, p. 1049-60, 1968. http://dx.doi.org/10.2307/4593479

MUSSELWHITE K. et al. The telephone interview is an effective method of data collection in clinical nursing research: a discussion paper. International Journal of Nursing Studies, London, v. 44, n. 6, p. 1064-70, 2007. PMid:16844128. http://dx.doi.org/10.1016/j. ijnurstu.2006.05.014

NAGELHOUT, G. E. et al. Is web interviewing a good alternative to telephone interviewing? Findings from the international tobacco control (ITC) Netherlands survey. BMC Public Health, London, v. 18, n. 10, p. 351, 2010.

PACHECO, W. et al. A era da tecnologia da informação e comunicação e a saúde do trabalhador. Revista Brasileira de Medicina do Trabalho, Belo Horizonte, v. 3, n. 2, p. 114-22, 2005.

PIAMJARIYAKUL, U.; BOTT, M. J.; TAUNTON, R. L. Strategies for an Internet-Based, Computer-Assisted 
Telephone Survey. Western Journal of Nursing Research, Beverly Hills, v. 28, n. 5, p. 602-609, 2006. PMid:16829640. http://dx.doi.org/10.1177/0193945906288582

PINTO-MEZA, A. et al. Assessing depression in primary care with the PHQ-9: Can it be carried out over the telephone? Journal of General Internal Medicine, Philadelphia, v. 20, n. 8, p. 738-42, 2005. PMid:16050884 PMCid:PMC1490180. http://dx.doi. org/10.1111/j.1525-1497.2005.0144.x

ROCHELEAU, C. M. et al. Effect of survey instrument on participation in a follow-up study: a randomization study of a mailed questionnaire versus a computer-assisted telephone interview. BMC Public Health, London, v. 31, n. 12, p. 579, 2012. PMid:22849754 PMCid:PMC3506531. http://dx.doi.org/10.1186/1471-2458-12-579

SCHWARTZ, L. M.; WOLOSHIN, S.; BIRKMEYER, J. D. How do elderly patients decide where to go for major surgery? Telephone interview survey. BMJ: British Medical Journal, London, v. 331, n. 7520, p. 821, 2005. PMid:16192286 PMCid:PMC1246083. http://dx.doi. org/10.1136/bmj.38614.449016.DE

SIKORSKII, A. et al. Differential symptom reporting by mode of administration of the assessment: automated voice response system versus a live telephone interview. Medical Care, Philadelphia, v. 47, n. 8, p. 866-74, 2009.
PMid:19584761 PMCid:PMC2722377. http://dx.doi. org/10.1097/MLR.0b013e3181a31d00

SMITH, E. M. Telephone interviewing in healthcare research: a summary of the evidence. Nursing Research, London, v. 12, n. 3, p.32-41, 2005.

STANLEY, D. Accessing elite nurses for research: reflections on the theoretical and practical issues of telephone interviewing. Journal of Research in Nursing, London, v. 13, n. 3, p. 236-248, 2008.

WARD-KING, J. et al. Brief report: telephone administration of the autism diagnostic interview-revised: reliability and suitability for use in research. Journal of Autism and Developmental Disorders, New York, v. 40, p. 1285-1290, 2010. PMid:20195733. http://dx.doi. org/10.1007/s10803-010-0987-x

WHITE, M. J. et al. Implementing a computer-assisted telephone interview (CATI) system to increase colorectal cancer screening: a process evaluation. Patient Education and Counseling, Limerick, v. 61, n. 3, p. 419-28, 2006. PMid:15993558. http://dx.doi.org/10.1016/j. pec.2005.05.008

WISE, M. et al. Integrating a narrative medicine telephone interview with online life review education for cancer patients: lessons learned and future directions. Journal of the Society for Integrative Oncology, Hamilton, v. 7, n. 1, p. 19-25, 2009.

\section{Contribuição dos Autores}

Gonçalo C. S. e Barros N. F. participaram igualmente de todas as etapas de elaboração do artigo.

\section{Notas}

${ }^{1}$ Este trabalho contou com financiamento Fapesp por meio do processo n. 2010/05217-0.

${ }^{2}$ As unidades de cuidados de longa duração oferecem uma variedade de serviços que abrange tanto as necessidades médicas e quanto as não-médicas de pessoas acometidas por doenças crônicas ou deficiência aque não podem cuidar de si mesmas por longos períodos de tempo.

${ }^{3} \mathrm{ADHD}=$ attention-deficit hyperactivity disorder, ou transtorno do déficit de atenção com hiperatividade (TDAH).

${ }^{4}$ Medicare é um programa de seguro social nacional, administrado pelo governo federal dos EUA desde 1965, que garante o acesso ao seguro de saúde para os americanos com idade acima de 65 anos, jovens com deficiência e pessoas com doença renal terminal.

${ }^{5}$ O recordatório alimentar é um método retrospectivo de avaliação dietética em que um indivíduo é entrevistado sobre o seu consumo de alimentos e bebidas durante um determinado período de tempo, normalmente o dia anterior ou as vinte e quatro horas precedentes.

${ }^{6}$ Practice development é um termo que abrange uma grande variedade de atividades, incluindo a introdução de mudanças na prática, o apoio e desenvolvimento de profissionais, o estabelecimento de padrōes e melhoria da qualidade. Ele é frequentemente usado livremente e de forma intercambiável com outros termos, tais como a implementação de evidências de pesquisa. 